\title{
Cationic Oligomer as an Organic Template for Direct Synthesis of Aluminosilicate ITH Zeolite
}

\author{
Chi Lei, ${ }^{+,}\left[{ }^{[a]}\right.$ Zhuoya Dong, ${ }^{+, ~[b]}$ Cristina Martínez, ${ }^{[c]}$ Joaquín Martínez-Triguero, ${ }^{[c]}$ Wei Chen, ${ }^{[d]}$ Qinming \\ Wu, ${ }^{*[a]}$ Xiangju Meng, ${ }^{\left[{ }^{[a]}\right.}$ Andrei-Nicolae Parvulescu, ${ }^{[\mathrm{e}]}$ Ulrich Müller, ${ }^{\left[{ }^{[e]}\right.}$ Anmin Zheng, ${ }^{\left[{ }^{[d]}\right.}$ Yanhang Ma, ${ }^{*[b]}$ \\ Weiping Zhang, ${ }^{[\mathrm{f}]}$ Toshiyuki Yokoi, ${ }^{[\mathrm{g}]}$ Bernd Marler, ${ }^{[\mathrm{h}]}$ Dirk E. De Vos, ${ }^{[i]}$ Ute Kolb, ${ }^{[i]}$ Avelino Corma*[c] and \\ Feng-Shou Xiao*[a]
}

\begin{abstract}
Despite great progress in the synthesis of aluminosilicate zeolites, there are still a large number of zeolites that could not be prepared in the aluminosilicate form. One of typical examples is ITH zeolite, where direct synthesis of aluminosilicate ITH is currently still challenging. Herein, for the first time we demonstrate the successful synthesis of aluminosilicate ITH zeolite using a simple cationic oligomer as an organic template. The key to this success is that the cationic oligomer has a strong complexation ability with aluminum species combined with a structural directing ability for the ITH structure similar to that of the conventional organic template. Physicochemical analysis shows that the aluminosilicate ITH zeolite has very high crystallinity, nanosheet-like crystal morphology, large surface area, fully four-coordinated Al species, and abundant acidic
\end{abstract}

[a] C. Lei, Dr. Q. Wu, Dr. X. Meng, Prof. F.-S. Xiao Key Laboratory of Applied Chemistry of Zhejiang Province Zhejiang University

Hangzhou 310007, P. R. China

E-mail: qinmingwu@zju.edu.cn; fsxiao@zju.edu.cn

[b] Z. Dong, Dr. Y. Ma

School of Physical Science and Technology

ShanghaiTech University

Shanghai 201210, P. R. China

E-mail: mayh2@shanghaitech.edu.cn

[c] Dr. C. Martínez, Dr. J. Martínez-Triguero, Prof. A. Corma Instituto de Tecnología Química

Universitat Politècnica de València-Consejo Superior de Investigaciones Científicas

Avenida de los Naranjos s/n, 46022 València, Spain

E-mail: acorma@itq.upv.es

[d] Dr. W. Chen, Prof. A. Zheng

Wuhan Institute of Physics and Mathematics, Chinese Academy of Sciences

Wuhan 430071, P. R. China.

[e] Dr. A.-N. Parvulescu, Dr. U. Müller

BASF SE, GCC/PZ-M311

Ludwigshafen 67056, Germany

[f] Prof. W. Zhang

State Key Laboratory of Fine Chemicals

Dalian University of Technology

Dalian 116012, P. R. China

[g] Prof. T. Yokoi

Chemical Resources Laboratory

Tokyo Institute of Technology

Yokohama 226-8503, Japan

[h] Prof. B. Marler

Department of Geology, Mineralogy and Geophysics

Ruhr University Bochum

Bochum 44780, Germany

[i] Prof. D. E. De Vos

Centre for Surface Chemistry and Catalysis

KU Leuven

Kasteelpark Arenberg 23, Leuven 3001, Belgium

[j] Prof. U. Kolb

Institute of Inorganic Chemistry and Analytical Chemistry

Johannes Gutenberg University Mainz

Mainz 555128, Germany

[+] These authors contributed equally to this work.

Supporting information for this article is given via a link at the end of the document.((Please delete this text if not appropriate)) sites. More importantly, methanol-to-propylene (MTP) tests reveal that the Al-ITH zeolite shows much higher selectivity for propylene and longer lifetime than commercial ZSM-5. FCC tests show that AlITH zeolite is a good candidate as a shape selective FCC additive for enhancing propylene and butylene selectivity.

\section{Introduction}

Zeolites, as a typical family of microporous materials, have shown important roles in the process of oil refining and fine chemicals production because of their uniform channel distribution, high surface area, and large micropore volume. ${ }^{[1-22]}$ As an important type of zeolites, germanosilicate-based zeolites provide many new structures, exhibiting excellent performance in various catalytic reactions. ${ }^{[23-34]}$ For example, BEC zeolite displays good catalytic performance in the isomerization of glucose to fructose; ${ }^{[24]}$ ECNU-21 zeolite is an efficient shape selective catalyst for ethylene oxide hydration; ${ }^{[25]} \mathrm{ITH}$ zeolite shows excellent performance in the catalytic cracking and methanol-topropylene (MTP) reaction. ${ }^{[26-29]}$

ITH zeolite, firstly discovered by Corma's group, ${ }^{[30]}$ has a special framework structure (three dimensional $9 \times 10 \times 10$ membered ring channels; aperture size of $4.0 \times 4.8,4.8 \times 5.1$, and $4.8 \times 5.3 \AA$ ). It could be prepared in the form of silicate and borosilicate but it was tough to synthesize the aluminosilicate form due to competitive growth of EUO when aluminum is present in the synthesis gel. ${ }^{[27,31-33]}$ In order to incorporate aluminum in the structure of ITH zeolite to extend the catalytic applications, the addition of germanium species in the ITH zeolite synthesis was always required. ${ }^{[26-29]}$ This addition not only greatly increases the synthetic cost, but also significantly reduces the hydrothermal and thermal stabilities of the ITH zeolite. ${ }^{[34]}$ The drawbacks mentioned above strongly limit its catalytic application. To avoid the use of germanium, a post-synthesis route was developed through alumination of borosilicate ITH zeolite into aluminosilicate ITH zeolite. ${ }^{[30]}$ Up to now, it was still a challenge to directly synthesize aluminosilicate ITH zeolite.

Recently, it has been reported that many zeolites such as $\mathrm{RHO}$, Beta, and GME could be successfully synthesized using cationic polymers/oligomers as novel organic templates. ${ }^{[35-37]}$ In these cases, it is worth noting that the zeolite products have more Al species in the framework than those synthesized from conventional organic templates. One possibility is that the cationic polymers/oligomers have a stronger complexation ability with aluminum species than conventional templates. Therefore, it is suggested that the use of novel cationic oligomer as an 

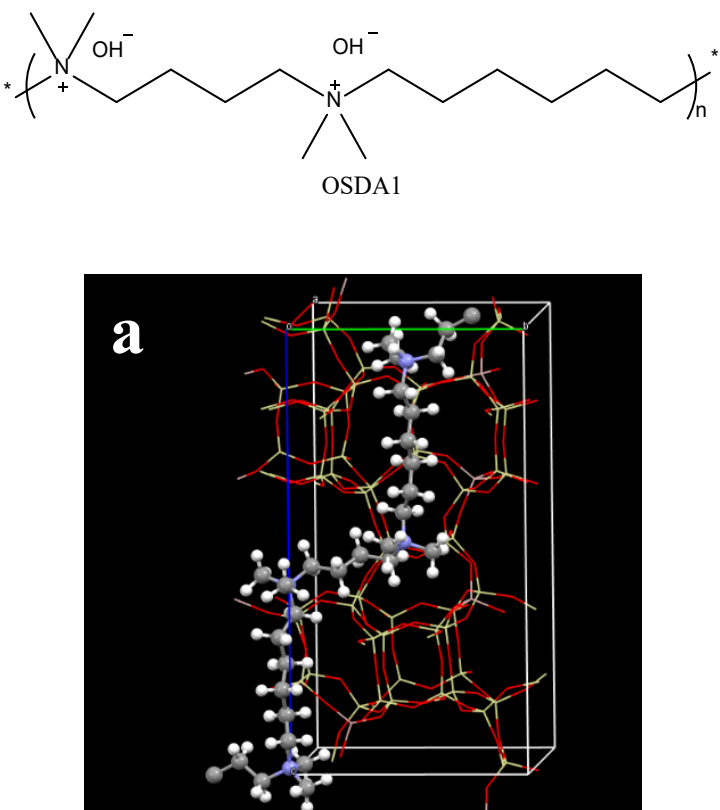

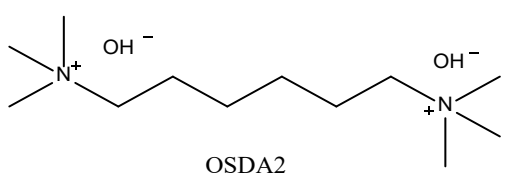

OSDA2

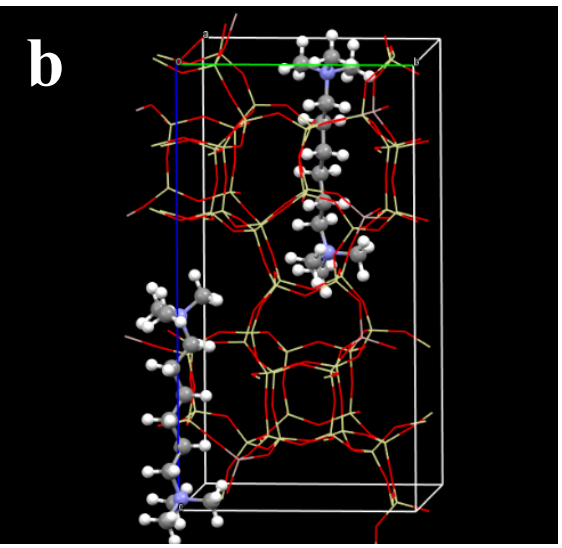

Figure 1 Structures of the cationic oligomer (OSDA1, a) and traditional template (OSDA2, b), and their positions within the all silica ITH zeolite framework

efficient organic template might be favourable for direct synthesis of the aluminosilicate ITH zeolite.

In this work, using a simple cationic oligomer as an organic template, the direct synthesis of an aluminosilicate ITH zeolite (AIITH) was shown to be successful. The obtained Al-ITH zeolite has a Si/Al ratio as low as 57 . More interestingly, the $\mathrm{Al}-\mathrm{ITH}$ shows excellent hydrothermal stability and good performance in both methanol-to-propylene (MTP) and FCC reactions.

\section{Results and Discussion}

In order to search for a suitable cationic oligomer for directing the ITH zeolite structure, theoretical simulations were performed, as presented in Figure 1 and Table S1. Notably, the interaction stability energy between the pure silica ITH zeolite framework and the structure of the cationic oligomer (Figure 1, OSDA1) is very similar to that of the conventional organic template (Figure 1, OSDA2), suggesting the possibility of the cationic oligomer for directing the ITH zeolite. In order to compare the complexation ability of the organic templates with aluminum species, both OSDA1 and OSDA2 solutions were mixed with a certain amount of $\mathrm{AlF}_{3}$. ICP analysis shows that the concentration of aluminum species in the solution of OSDA1 is much higher than that of the OSDA2, as presented in Table S2. These results indicate that the OSDA1 has indeed stronger complexation ability with aluminum species than the OSDA2. To measure the importance of $\mathrm{Al}$ complexation ability for OSDA1, we performed additional experiments for addition of aluminum source $\left(\mathrm{AlF}_{3}\right)$ in the final gel $\left(\mathrm{H}_{2} \mathrm{O} / \mathrm{SiO}_{2}\right.$ at 3$)$ by partial removal of water from the starting gel $\left(\mathrm{H}_{2} \mathrm{O} / \mathrm{SiO}_{2}>30\right)$. In the final gel, the complexation ability between OSDA1 with aluminum species should be weak due to shortage of water solution, compared with the starting gel. As a result, the addition of aluminum species in the final gel leads to the formation of amorphous product rather than the Al-ITH zeolite (Figure S1), confirming the importance of the complexation of Al with OSDA1. Therefore, both structural directing ability and strong complexation ability with aluminum species of the OSDA are very important for the successful synthesis of Al-ITH zeolite.

Figure $2 \mathrm{~A}$ shows $\mathrm{X}$-ray diffraction $(\mathrm{XRD})$ pattern of as-made Al-ITH zeolite from the initial gel with Si/Al ratio at 100 , exhibiting a series of characteristic peaks associated with ITH structure (Si/Al ratio of 70 ), in good agreement with the simulated XRD pattern (Figure S2). The scanning electron microscopy (SEM) images in Figure 2B and Figure $\mathrm{S} 3$ show that the as-made $\mathrm{Al}$ ITH zeolite has a nanosheet-like morphology with a thickness of about $30 \mathrm{~nm}$. Figure 2C displays nitrogen sorption isotherm of calcined AI-ITH zeolite, giving BET surface area and micropore volume of about $400 \mathrm{~m}^{2} / \mathrm{g}$ and $0.15 \mathrm{~cm}^{3} / \mathrm{g}$, respectively. In addition, the measured external surface area of $64 \mathrm{~m}^{2} / \mathrm{g}$ and mesopore volume of $0.22 \mathrm{~cm}^{3} / \mathrm{g}$, might be related to the nanosheet-like morphology of Al-ITH zeolite. Furthermore, Si/Al ratio of Al-ITH zeolite is determined to be 70 by inductively coupled plasma (ICP) technique. Figure $2 \mathrm{D}$ shows ${ }^{29} \mathrm{Si}$ MAS NMR spectrum of as-made Al-ITH zeolite, giving four peaks with the chemical shift centered at $-116.8,-114.2,-111.3$, and -105.4 

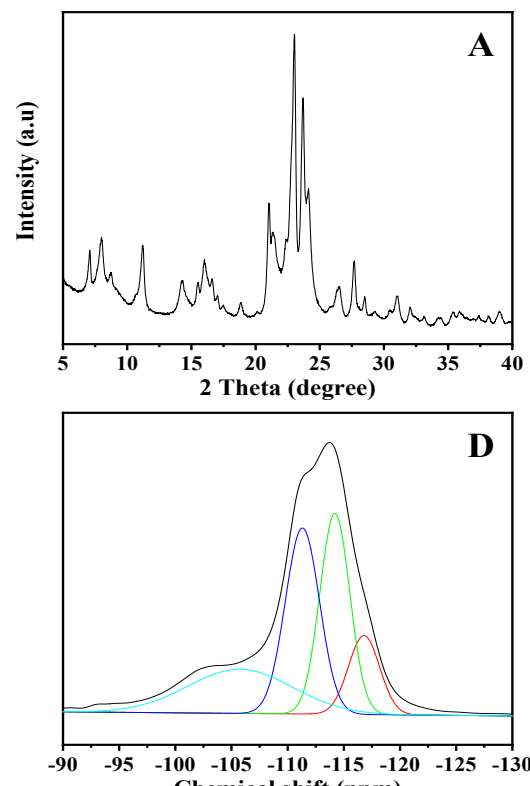

Chemical shift (ppm)

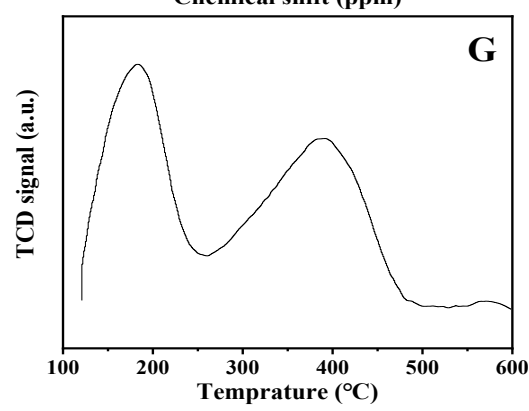

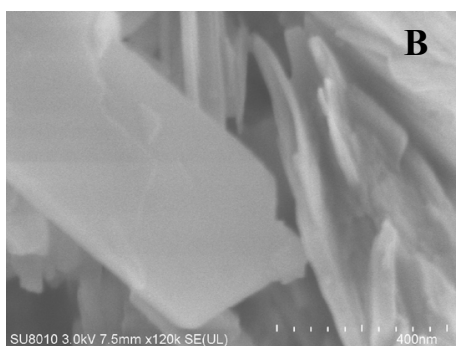
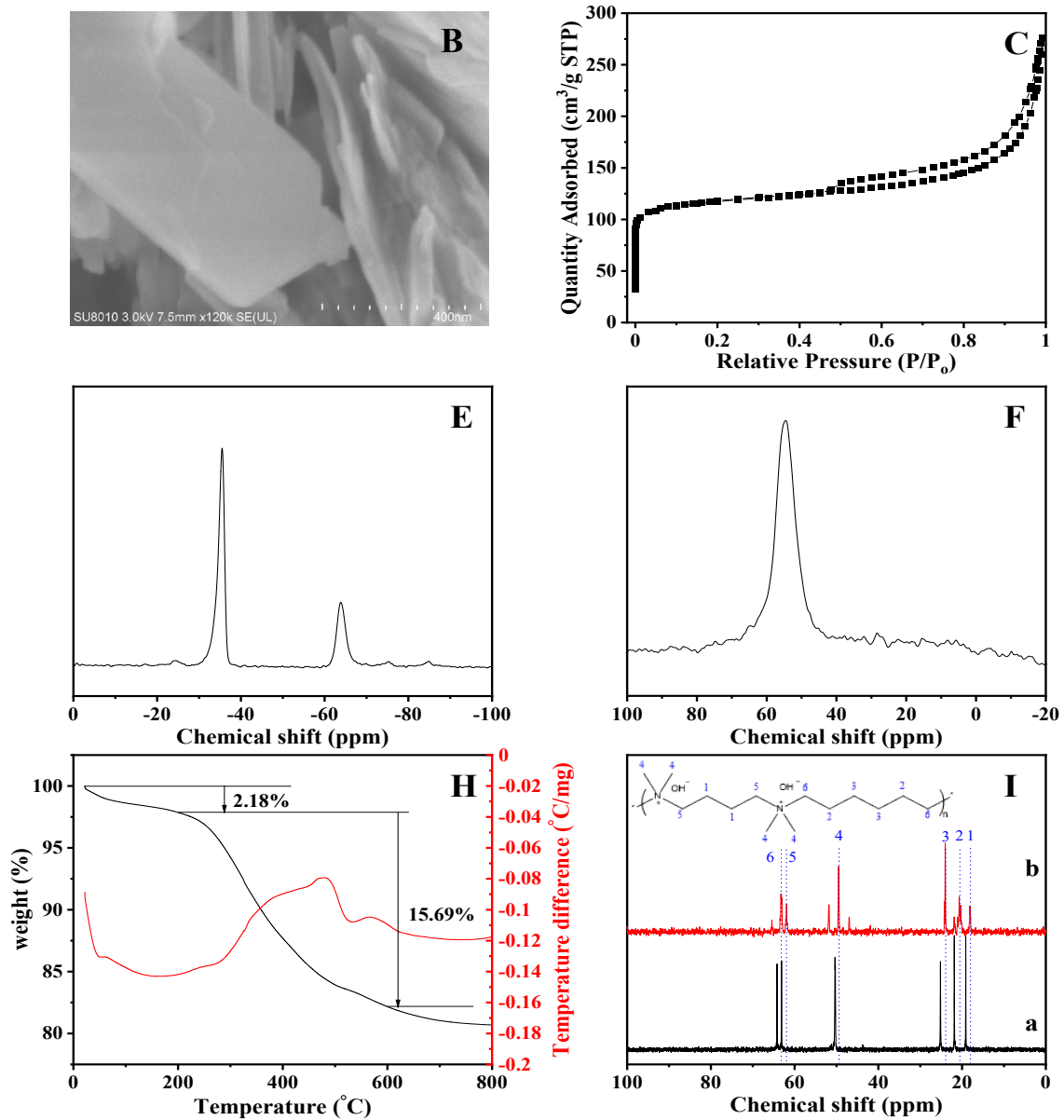

Figure 2 (A) XRD pattern (wavelength: $1.5406 \AA$ ), (B) SEM image, (C) $\mathrm{N}_{2}$ sorption isotherm, (D) ${ }^{29} \mathrm{Si}$ MAS NMR, (E) ${ }^{19} \mathrm{~F}$ MAS NMR, (F) ${ }^{27} \mathrm{Al}$ MAS NMR, (G) NH ${ }_{3}^{-}$ TPD curve, (H) TG-DTA curve of Al-ITH zeolite product; and (I) ${ }^{13} \mathrm{C}$ NMR of a) template solution and b) template dissolved from Al-ITH sample.

ppm. The first three peaks are assigned to $\mathrm{Si}(4 \mathrm{Si})$ species, and the fourth one is attributed to the $\mathrm{Si}(3 \mathrm{Si}, \mathrm{OH})$ and/or $\mathrm{Si}(3 \mathrm{Si}, 1 \mathrm{Al})$. Figure $2 \mathrm{E}$ exhibits ${ }^{19} \mathrm{~F}$ MAS NMR spectrum of as-made Al-ITH zeolite, displaying two peaks at -35.5 and $-63.8 \mathrm{ppm}$, which are assigned to fluoride species in the double four member rings and the $\left[4^{1} 5^{2} 6^{2}\right]$ cage, respectively. ${ }^{[33]}$ Figure $2 \mathrm{~F}$ gives ${ }^{27} \mathrm{Al}$ MAS NMR spectrum of as-made Al-ITH zeolite, showing a peak at $53.3 \mathrm{ppm}$ associated with four-coordinated aluminum species in the ITH zeolite framework. We cannot observe the peak at round $0 \mathrm{ppm}$, implying the absence of six-coordinated aluminum species in the Al-ITH zeolite product. Figure $2 \mathrm{G}$ shows temperature programmed desorption of ammonia $\left(\mathrm{NH}_{3}\right.$-TPD) curve of the $\mathrm{H}$ Al-ITH zeolite, showing two desorption peaks centered at about $185^{\circ} \mathrm{C}$ and $390^{\circ} \mathrm{C}$, which are very similar to those of Al-Ge-ITH zeolite synthesized by direct method reported in the literatures. ${ }^{[28]}$ Thermal analysis (TG-DTA, Figure2H) of as-made Al-ITH zeolite shows a weight loss of $15.69 \%$ in the region of $200-600{ }^{\circ} \mathrm{C}$, which is attributed to the decomposition of OSDA1. ${ }^{13} \mathrm{C}$ nuclear magnetic resonance (NMR) spectrum of the liquid phase obtained by dissolving as-made Al-ITH zeolite with a dilute HF solution is close to that of the OSDA1 solution (Figure 2I). In addition, many weak peaks have been observed in the dissolved solution treated from Al-ITH zeolite, which could be related to the Hoffman elimination of a small amount of OSDA1 at relatively high temperature (Figure S4). Combining with the results of NMR and TG-DTA characterizations, it is concluded that the OSDA1 mainly exists in the micropores of the Al-ITH zeolite.

Figure 3 shows selected-area electron diffraction (SAED) patterns and transmission electron microscopy (TEM) images of as-synthesized Al-ITH zeolite. Figure 3a-3c are collected from a nanosheet-like crystal lying flat on the grid. The indexing of SAED pattern with sharp diffraction spots and comparison of experimental and simulated high-resolution TEM images indicate that the crystal has a preferred orientation along the $c$ axis. Another crystal standing vertically on the grid was aligned to the [100] zone axis (Figure 3d-f). The TEM image viewed along the a axis shows that the thickness of the crystal is around $30 \mathrm{~nm}$, in good agreement with that of SEM images. Both projections along these two zone axes match well with the simulated images using 
the ITH zeolite framework (Figures S5-S7). These results confirm the successful synthesis of Al-ITH zeolite with ITH structure in the presence of the novel cationic oligomer.
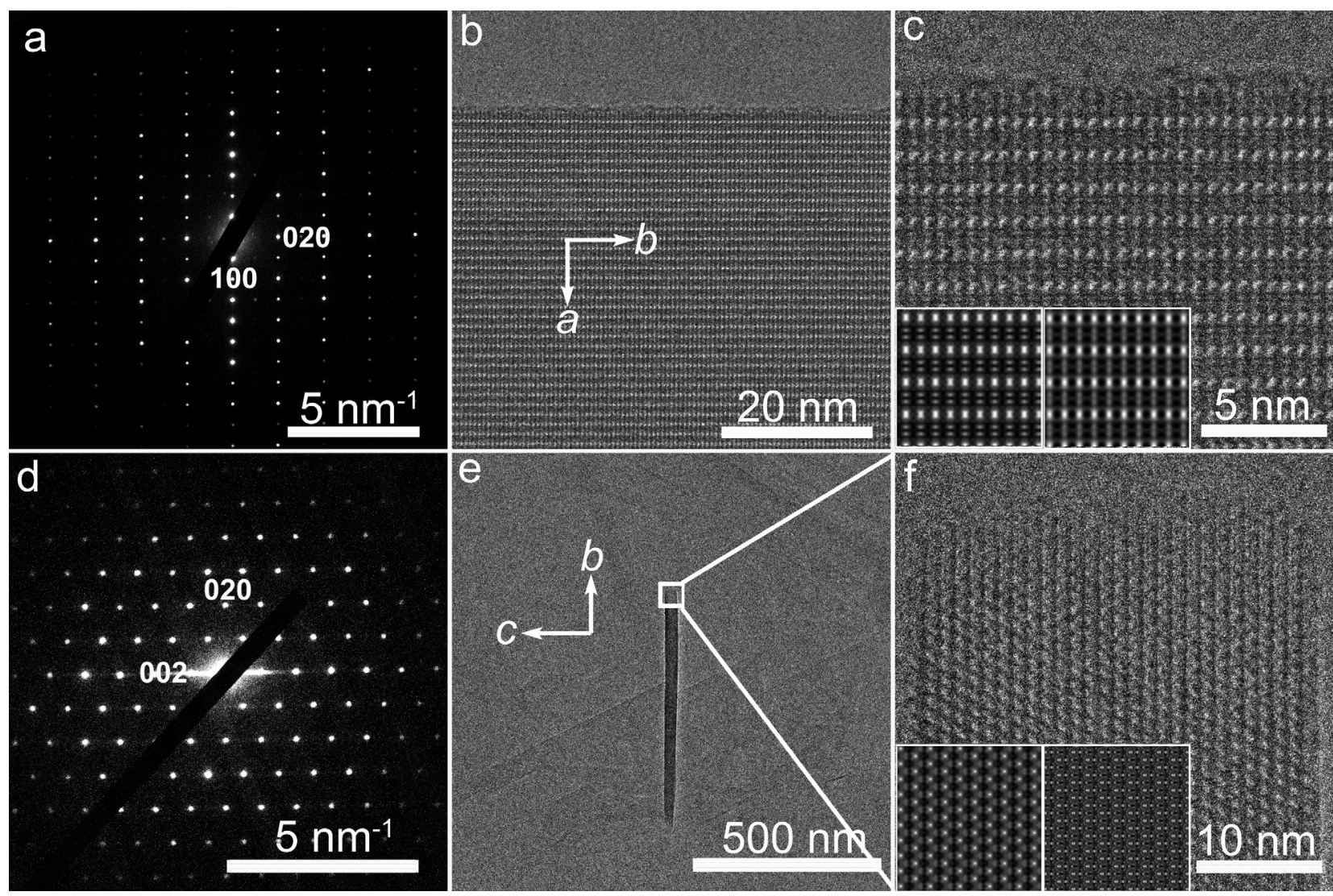

Figure 3 SAED patterns and TEM images of Al-ITH zeolite viewed along the [001] (a, b and c) and [100] (d, e and f) zone axes. The insets in (c) and (f) are symmetry-averaged (left) and simulated images (right), respectively. The settings of defocus values and thickness for (c) and (f) are $500 \mathrm{~nm} \& 5.2 \mathrm{~nm}$ and $-120 \mathrm{~nm}$ \& $2.6 \mathrm{~nm}$, respectively.
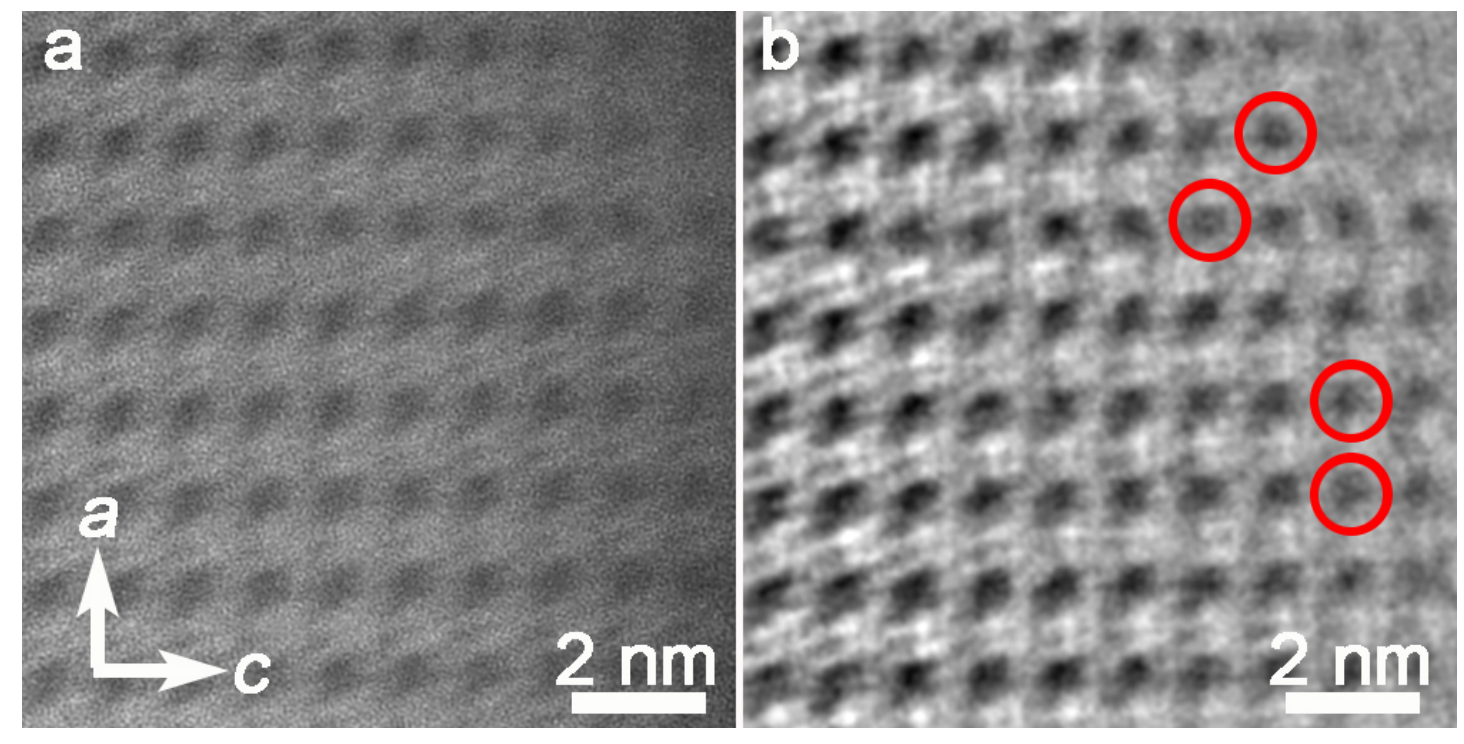

Figure 4 HAADF-STEM (a) and qDPC-STEM (b) images of as-synthesized Al-ITH zeolite viewed along [010] zone axis. Circles on Figure 4b with the white contrast spots might represent organic templates molecules inside the zeolite channel. 
Quantitative differential phase contrast (qDPC) has emerged as an effective approach to study e-beam sensitive porous materials using scanning transmission electron microscopy (STEM) mode. ${ }^{[38,39]}$ Compared with high-angle annular dark field (HAADF) image, qDPC-STEM shows advantages on visualizing both heavy and light elements at the same time.$^{[40-45]}$ As a result, it is possible to obtain information on organic templates in the AlITH zeolite micropores using qDPC-STEM images. From the structure model of ITH zeolite (Figure S5), straight channels of 10 MR and 9 MR ring would be clearly seen along [010] and [100] zone axes, respectively. Therefore, qDPC-STEM images should be taken along these two directions to observe organic templates. However, Al-ITH zeolite is a nanosheet-like material and [001] zone axis is vertical to the plate, which means that the Al-ITH crystals along [010] or [100] zone axis would be difficult to find otherwise it is too thick to take qDPC-STEM images clearly. Fortunately, we were able to find a crystal with the thin section along [010] zone axis and thus obtain the HAADF-STEM and qDPC-STEM images of Al-ITH zeolite, as shown in Figure 4. Compared with HAADF image (Figure 4a) without the observation of any organic template due to the limitation of HAADF technique, it would be clearly seen that there are some qDPC-STEM image (Figure 4b), which might be related to organic template molecules occluded in the zeolite micropore channels. Combining with the result of theoretical simulation, NMR and TG-DTA characterizations, and qDPC-STEM images, it would further be concluded that the OSDA1 molecule, which is fully positioned in the micropore of aluminosilicate Al-ITH zeolite, is indeed an organic template for directing the formation of aluminosilicate $\mathrm{Al}$ ITH zeolite structure.

To understand the Al-ITH crystallization, XRD, SEM, and MAS NMR characterization techniques were used. Before the crystallization, only an amorphous phase is observed (Figure S8a and Figure S9a). After 4 days of crystallization, the XRD pattern of the sample shows very weak peaks associated with the Al-ITH zeolite (Figure S8b). At the same time, the Al-ITH zeolite crystals with nanosheet-like morphology can clearly be seen from the SEM image (Figure S9b). Increasing the crystallization time from 5 to 8 days, the XRD peak intensity of the samples gradually increases (Figures S8c-8f). Correspondingly, more Al-ITH zeolite crystals are observed in the SEM images (Figures S9c-9f). After 10 days, Al-ITH zeolite is obtained with high crystallinity (Figure S8g and Figure S9g). The dependence of the Al-ITH zeolite crystallinity on the crystallization time is shown in Figure $\mathrm{S} 10$. Figures S11 and S12 show ${ }^{27} \mathrm{Al}$ and ${ }^{29} \mathrm{Si}$ MAS NMR spectra of AlITH zeolite products obtained after the different crystallization times. As shown in Figure S11, the products after 4 to 10 days of crystallization have a peak near 60 ppm associated with 4coordinated aluminum species, while the aluminum species are 6 -coordinated at the beginning in the crystallization. These results show that 6-coordinated aluminum species have been completely transferred into 4-coordinated species in the initial stage of the crystallization. As shown in Figure S12 and Tables S3-S4, the maximum percentage of $\mathrm{Si}(3 \mathrm{Si})$ species $(-101.9 \mathrm{ppm})$ appears at 5 days. Correspondingly, the XRD data show the crystallization happening fast at this stage. Possibly, the rearrangement of silicon species with the intermediate of $\mathrm{Si}(3 \mathrm{Si})$ under assistance of organic templates plays a critical role in the crystallization.
It is well known that high hydrothermal stability is a key factor for many industrial applications of zeolites. ${ }^{[46,47]}$ Therefore, it is very crucial to investigate the hydrothermal stability of Al-ITH zeolite in this work. After steaming treatment at $800{ }^{\circ} \mathrm{C}$ for $5 \mathrm{~h}$ with $10 \%$ water, the crystallinity, surface area, and pore volume of aged $\mathrm{H}$-Al-ITH zeolite are very close to those of the fresh one (Figure S13, Tables S5-S6). These results demonstrate that the Al-ITH zeolite has extraordinarily hydrothermal stability, which would be favorable for its industrial application.

Figure 5 and Figure S14 shows the catalytic performances in the MTP reaction over Al-ITH-70, Al-ITH-94, SiGeAl-ITH, SiAl(B)ITH, and ZSM-5 zeolites. Table S7 presents the catalytic data after $2 \mathrm{~h}$ time on stream. Obviously, the Al-ITH zeolites combine the advantages of high selectivity for propylene and long catalytic lifetime among the tested zeolite samples. In addition, it is worth noting that the absence of Ge species would stabilize the structure of Al-ITH zeolite against the irreversible hydrothermal effect of the water produced in the MTO reaction. Therefore, the above results are potentially important for selective production of propylene in the industrial applications.

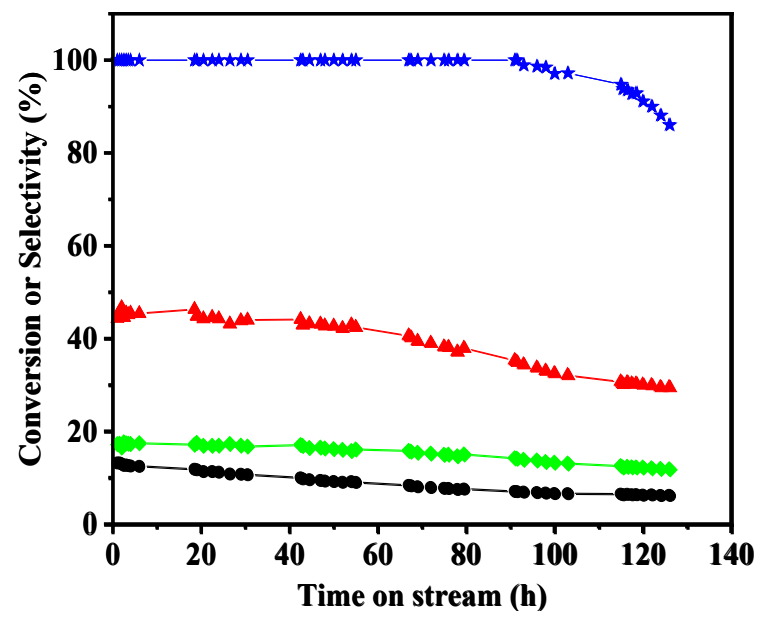

Figure 5 Dependences of methanol conversion and product selectivity on reaction time in MTP reaction over the Al-ITH zeolite at $480{ }^{\circ} \mathrm{C}$. O: $\mathrm{C}_{2}=$ selectivity, $\boldsymbol{\Delta}: \mathrm{C}_{3}{ }{ }^{2}$ selectivity, $\diamond: \mathrm{C}_{4}{ }^{=}$selectivity, $\star$ : Conversion of methanol.

In addition, it has been reported that the structural and acidic properties of Al-ITH make it a good alternative to ZSM- 5 as shape selective FCC additive for producing propylene. ${ }^{[27,48]}$ Thus, the catalytic behaviour of the AI-ITH sample and two ZSM- 5 samples have been compared for catalytic cracking of a vacuum gasoil (VGO, see Table S8 for VGO properties) in a microactivity test unit. ${ }^{[28]}$ The results are shown in Figures S15-S17. The presence of the additives results in all cases in a decrease of the gasoline fraction and an increase of the gases produced due to cracking of the olefins and paraffins present in the gasoline. The drop in gasoline yield is higher for the ZSM- 5 additive with the highest Al content and lowest for the H-Al-ITH. Diesel and coke selectivity are not affected. The highlight is the Al-ITH additive increases propylene yield to levels close to those obtained with ZSM-5 (113) but without increasing the yield of propane. Moreover, it also increases the yield to the linear butenes to values comparable to 
those given by the ZSM-5 zeolites with no increase of the saturated butanes. This results in higher olefin to paraffin ratios in the $\mathrm{C}_{3}$ and $\mathrm{C}_{4}$ fractions. When comparing the catalytic behaviour of $\mathrm{Al}-\mathrm{ITH}$ with that of a $\mathrm{GeAl}-\mathrm{ITH}(\mathrm{Si} / \mathrm{Ge}=10,(\mathrm{Si}+\mathrm{Ge}) / \mathrm{Al}=50)$ described previously ${ }^{[27]}$ it can be seen that the latter, with a higher Al content, produces more gases by gasoline and even diesel cracking (see Figure S18a). However, Al-ITH, although yielding less propene, presents comparable propene/propane ratio (see Figure S18b) and the additional advantage of its higher thermal and hydrothermal stability due to the absence of Ge. Therefore, the above results show that Al-ITH zeolite is indeed a good candidate as an FCC additive. Therefore, the above results show that AI-ITH zeolite is indeed a good candidate as an FCC additive.

After the successful synthesis of Al-ITH zeolite with a Si/Al ratio of 70 in the final product (Table S9, Run 1), Al-ITH zeolites with various $\mathrm{Si} / \mathrm{Al}$ ratios $(57,94$, and 120$)$ are also synthesized (Table S9, Run 2-4). All products have a good crystallinity, fourcoordinated aluminum species, and crystals with nanosheet-like morphology (Figures S19-S21). The details for all the synthesis process are presented in Table S9.

\section{Conclusion}

In summary, the direct synthesis of Al-ITH zeolite with different $\mathrm{Si} / \mathrm{Al}$ ratios is successful in the presence of a cationic oligomer as an organic template. The key to the successful synthesis is the synergistic feature of the cationic oligomer with both the structural directing ability for crystallization of ITH zeolite and the strong complexation ability of aluminum species. The obtained Al-ITH zeolite has superior hydrothermal stability. Catalytic tests in MTP reaction show that the Al-ITH zeolite is active and selective for the production of propylene together with a long catalytic lifetime. In addition, Al-ITH zeolite is a good candidate as a shape selective FCC additive. The use of the cationic oligomers might open an alternative way in the future to synthesize other zeolites directly that up to now could not yet be crystallized as aluminosilicates.

\section{Experimental Section}

As a typical example for the organic template synthesis, $17.231 \mathrm{~g}$ of $\mathrm{N}, \mathrm{N}, \mathrm{N}$ ',N'-tetramethylhexane-1,6-diamine $(0.1 \mathrm{~mol})$ was mixed with 21.591 $\mathrm{g}$ of 1,4 -dibromobutane $(0.1 \mathrm{~mol})$ and $50 \mathrm{~mL}$ of ethanol. The mixture was put under reflux for $12 \mathrm{~h}$. Then, the solvent was evaporated, and the white sediment was washed with ether and dried under vacuum. Gel permeation chromatography (GPC) analysis showed that the bromide salt form of the template had a molecular weight between 4291 and $8669(n=11-22)$. The bromide form was converted to the hydroxide form using an anion exchange resin (IRN-78) in water, and the obtained solution was titrated using $0.1 \mathrm{M} \mathrm{HCl}$.

As a typical run for synthesis of Al-ITH zeolite, boehmite (0-24 mg, 0-0.16 $\mathrm{mmol} \mathrm{Al}_{2} \mathrm{O}_{3}$ ) and $10.67 \mathrm{~g}$ of organic template solution (OSDA1, $0.36 \mathrm{~mol} / \mathrm{L}$ $\mathrm{OH}^{-}$) were mixed. Then, $0.935 \mathrm{~g}$ of fumed silica $(15.58 \mathrm{mmol})$ was added under stirring. After stirring the mixture for another $6 \mathrm{~h}, 0.05 \mathrm{~g}$ of siliceous ITH zeolite seeds and $0.190 \mathrm{~mL}$ of HF $(40 \%, 3.3 \mathrm{mmol})$ were added. After partial water evaporation from starting gel $\left(\mathrm{H}_{2} \mathrm{O} / \mathrm{SiO}_{2}>30\right)$, the final molar composition of the mixture was $1.0 \mathrm{SiO}_{2}: 0-0.01 \mathrm{Al}_{2} \mathrm{O}_{3}: 0.005$ OSDA1 : $0.2 \mathrm{HF}: 1-3 \mathrm{H}_{2} \mathrm{O}$. Finally, the mixture was transferred into a Teflon-lined autoclave and heated at $160{ }^{\circ} \mathrm{C}$ for 10 days under rotation condition (50 $\mathrm{rpm}$ ). The solid zeolite product, which was denoted as Al-ITH-x, where $\mathrm{x}$ was the Si/Al ratios in the starting gel, was then filtered, washed with deionized water, dried at $100^{\circ} \mathrm{C}$ and calcined at $550{ }^{\circ} \mathrm{C}$ for $5 \mathrm{~h}$ and named as $\mathrm{H}$-Al-ITH-x. After hydrothermal treatment of $\mathrm{H}-\mathrm{Al}-\mathrm{ITH}$ zeolite samples at $800{ }^{\circ} \mathrm{C}$ with $10 \% \mathrm{H}_{2} \mathrm{O}$ for $5 \mathrm{~h}$, the aged zeolite product was obtained.

To evaluate the complexation ability of organic templates with aluminum species, $0.05 \mathrm{~g} \mathrm{AlF}_{3}$ and $10.67 \mathrm{~g}$ of template solution $\left(0.36 \mathrm{~mol}^{\prime} \mathrm{L} \mathrm{OH}^{-}\right.$in OSDA1 and OSDA2 aqueous solution) were mixed at room temperature. After stirring for $30 \mathrm{~min}$, the $\mathrm{Al}$ concentration of the supernatant was analysed using inductively coupled plasma (ICP). Considering the $\mathrm{AlF}_{3}$ is not soluble in water and does not react with base, if the supernatant has higher Al species concentration, it means the better complexation with $\mathrm{Al}$ species.

\section{Acknowledgements}

This work is supported by National Key Research and Development Program of China (2017YFB0702803), National Natural Science Foundation of China (21703203, 21673205, 21835002 and 21875140), Shanghai Pujiang Program (17PJ1406400) and the Young Elite Scientist Sponsorship Program by CAST (2017QNRC001). This work is partially supported by ChEM, SPST, ShanghaiTech (Grant 02161943), the Spanish Government-MICINN through "Severo Ochoa" (SEV 2012-0267) and RTI2018-101033-B-I00, and the European Union through ERC-AdG-2014-671093 (SynCatMatch). This work was also supported by BASF INCOE program.

Keywords: zeolite $\cdot$ aluminosilicate ITH • cationic oligomer • direct synthesis $\cdot$ MTP reaction

[1] J. Shin, D. Jo, S. B. Hong, Acc. Chem. Res. 2019, 52, 1419-1427.

[2] P. Tian, Y. Wei, M. Ye, Z. Liu, ACS Catal. 2015, 5, 1922-1938.

[3] F. Liu, Y. Yu, H. He, Chem. Commun. 2014, 50, 8445-8463.

[4] M. Dusselier, M. E. Davis, Chem. Rev. 2018, 118, 5265-5329.

[5] V. Vattipalli, A. M. Paracha, W. Hu, H. Chen, W. Fan, Angew. Chem. Int. Ed. 2018, 57, 3607-3611.

[6] Z. Liu, T. Wakihara, K. Oshima, D. Nishioka, Y. Hotta, S. P. Elangovan, Y. Yanaba, T. Yoshikawa, W. Chaikittisilp, T. Matsuo, T. Takewaki, T. Okubo, Angew. Chem. Int. Ed. 2015, 54, 5683-5687.

[7] Q. Sun, N. Wang, R. Bai, X. Chen, J. Yu, J. Mater. Chem. A 2016, 4, 14978-14982.

[8] X. Zhu, J. P. Hofmann, B. Mezari, N. Kosinov, L. Wu, Q. Qian, B. M. Weckhuysen, S. Asahina, J. Ruiz-Martínez, E. J. M. Hensen, ACS Catal. 2016, 6, 2163-2177.

[9] R. Li, N. Linares, J. G. Sutjianto, A. Chawla, J. Garcia - Martinez, J. D. Rimer, Angew. Chem. Int. Ed. 2018, 57, 11283-11288.

[10] X. Liu, H. Xu, L. Zhang, L. Han, J. Jiang, P. Oleynikov, L. Chen, P. Wu, ACS Catal. 2016, 6, 8420-8431.

[11] D. Xie, L. B. McCusker, C. Baerlocher, S. I. Zones, W. Wan, X. Zou, J. Am. Chem. Soc. 2013, 135, 10519-10524.

[12] D. W. Fickel, E. D'Addio, J. A. Lauterbach, R. F. Lobo, Appl. Catal. B Environ. 2011, 102, 441-448.

[13] M. T. Conato, M. D. Oleksiak, B. P. McGrail, R. K. Motkuri, J. D. Rimer, Chem. Commun. 2015, 51, 269-272.

[14] D. Jo, J. Bin Lim, T. Ryu, I.-S. Nam, M. A. Camblor, S. Bong Hong, J. Mater. Chem. A 2015, 3, 19322-19329.

[15] M. Choi, K. Na, J. Kim, Y. Sakamoto, O. Terasaki, R. Ryoo, Nature 2009 $461,246-249$ 
[16] Q. Wu, X. Meng, X. Gao, F.-S. Xiao, Acc. Chem. Res. 2018, 51, 13961403.

[17] A. Rojas, E. Martínez-Morales, C. M. Zicovich-Wilson, M. A. Camblor, J. Am. Chem. Soc. 2012, 134, 2255-2263.

[18] C. Li, M. Moliner, A. Corma, Angew. Chem. Int. Ed. 2018, 57, 1533015353.

[19] B. W. Boal, J. E. Schmidt, M. A. Deimund, M. W. Deem, L. M. Henling S. K. Brand, S. I. Zones, M. E. Davis, Chem. Mater. 2015, 27, 77747779.

[20] E.-P. Ng, D. Chateigner, T. Bein, V. Valtchev, S. Mintova, Science 2012 335, 70-73.

[21] V. Valtchev, L. Tosheva, Chem. Rev. 2013, 113, 6734-6760.

[22] N. Funase, T. Tanigawa, Y. Yamasaki, N. Tsunoji, M. Sadakane, T. Sano, J. Mater. Chem. A 2017, 5, 19245-19254.

[23] A. Corma, M. J. Díaz-Cabañas, J. Martínez-Triguero, F. Rey, J. Rius, Nature 2002, 418, 514-517.

[24] G. Zhang, P. Feng, W. Zhang, H. Liu, C. Wang, H. Ma, D. Wang, Z. Tian, Microporous Mesoporous Mater. 2017, 247, 158-165.

[25] X. Liu, W. Mao, J. Jiang, X. Lu, M. Peng, H. Xu, L. Han, S. Che, P. Wu, Chem. Eur. J. 2019, 25, 4520-4529.

[26] P. Zeng, X. Guo, X. Zhu, Q. Guo, Y. Wang, S. Ren, B. Shen, Microporous Mesoporous Mater. 2017, 246, 186-192.

[27] R. Castaneda, A. Corma, V. Fornes, J. Martineztriguero, S. Valencia, J. Catal. 2006, 238, 79-87.

[28] L. Li, Y. Chen, S. Xu, J. Li, M. Dong, Z. Liu, H. Jiao, J. Wang, W. Fan, J. Catal. 2016, 344, 242-251.

[29] H. Ma, Y. Chen, S. Wang, Z. Wei, Z. Qin, M. Dong, J. Li, W. Fan, J. Wang, Catal. Sci. Technol. 2018, 8, 521-533.

[30] A. Corma, M. Puche, F. Rey, G. Sankar, S. J. Teat, Angew. Chem. Int Ed. 2003, 42, 1156-1159.

[31] G. Xu, X. Zhu, X. Li, S. Xie, S. Liu, L. Xu, Microporous Mesoporous Mater 2010, 129, 278-284.

[32] X. Liu, U. Ravon, A. Tuel, Microporous Mesoporous Mater. 2012, 156 257-261.

[33] Q. Wu, X. Liu, L. Zhu, X. Meng, F. Deng, F. Fan, Z. Feng, C. Li, S. Maurer, M. Feyen, U. Müller, F.-S. Xiao, Chin. J. Chem. 2017, 35, 572-576.

[34] A. Corma, M. E. Davis, ChemPhysChem 2004, 5, 304-313.

[35] S. Liu, P. Zhang, X. Meng, D. Liang, N. Xiao, F.-S. Xiao, Microporous Mesoporous Mater. 2010, 132, 352-356.

[36] R. H. Daniels, G. T. Kerr, L. D. Rollmann, J. Am. Chem. Soc. 1978, 100 3097-3100.

[37] J. Zhu, Y. Zhu, L. Zhu, M. Rigutto, A. van der Made, C. Yang, S. Pan, L. Wang, L. Zhu, Y. Jin, Q. Sun, Q. Wu, X. Meng, D. Zhang, Y. Han, J. Li, Y. Chu, A. Zheng, S. Qiu, X. Zheng, F.-S. Xiao, J. Am. Chem. Soc. 2014 136, 2503-2510.

[38] B. Shen, X. Chen, D. Cai, H. Xiong, X. Liu, C. Meng, Y. Han, F. Wei, Adv. Mater. 2020, 32, 1906103.

[39] L. Liu, N. Wang, C. Zhu, X. Liu, Y. Zhu, P. Guo, L. Alfilfil, X. Dong, D. Zhang, Y. Han, Angew. Chem. Int. Ed. 2020, 132, 829-835.

[40] N. Shibata, Y. Kohno, S. D. Findlay, H. Sawada, Y. Kondo, Y. Ikuhara, J. Electron Microsc. (Tokyo) 2010, 59, 473-479.

[41] N. Shibata, S. D. Findlay, Y. Kohno, H. Sawada, Y. Kondo, Y. Ikuhara, Nat. Phys. 2012, 8, 611-615.

[42] E. Yücelen, I. Lazić, E. G. T. Bosch, Sci. Rep. 2018, 8, 1-10.

[43] I. Lazić, E. G. T. Bosch, S. Lazar, Ultramicroscopy 2016, 160, 265-280.

[44] A. Ishizuka, M. Oka, T. Seki, N. Shibata, K. Ishizuka, Microscopy 2017, 66, 397-405.

[45] A. Ishizuka, M. Oka, K. Ishizuka, T. Seki, N. Shibata, Microsc. Microanal. 2017, 23, 34-35.

[46] T. Ryu, N. H. Ahn, S. Seo, J. Cho, H. Kim, D. Jo, G. T. Park, P. S. Kim, C. H. Kim, E. L. Bruce, P. A. Wright, I.-S. Nam, S. B. Hong, Angew. Chem Int. Ed. 2017, 56, 3256-3260.

[47] L. Zhu, L. Ren, S. Zeng, C. Yang, H. Zhang, X. Meng, M. Rigutto, A. van der Made, F.-S. Xiao, Chem. Commun. 2013, 49, 10495-10497.

[48] A. I. Hussain, A. Palani, A. M. Aitani, J. Čejka, M. Shamzhy, M. Kubŭ, S. S. Al-Khattaf, Fuel Process. Technol. 2017, 161, 23-32. 


\section{Entry for the Table of Contents}

\section{RESEARCH ARTICLE}

This work shows a way to directly synthesize aluminosilicate ITH (AlITH) zeolite using a cationic oligomer as an organic template, where the strong complexation ability with aluminum species is a key factor for the successful preparation.

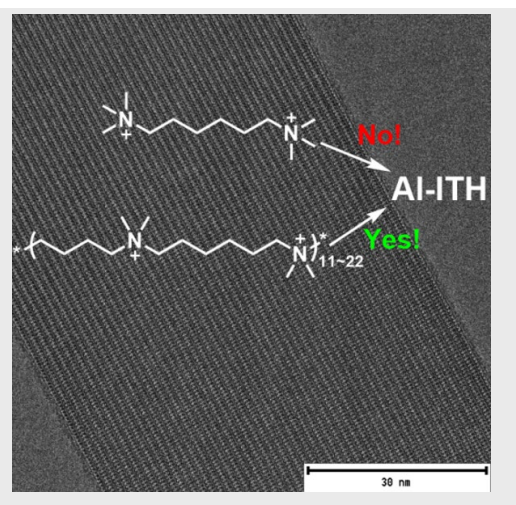

Chi Lei, Zhuoya Dong, Cristina Martínez, Joaquín Martínez-Triguero, Wei Chen, Qinming $W u,{ }^{*}$ Xiangju Meng, AndreiNicolae Parvulescu, Ulrich Müller, Anmin Zheng, Yanhang Ma, ${ }^{*}$ Weiping Zhang, Toshiyuki Yokoi, Bernd Marler, Dirk E. De Vos, Ute Kolb, Avelino Corma* and Feng-Shou Xiao*

Page No. - Page No.

Cationic Oligomer as an Organic Template for Direct Synthesis of Aluminosilicate ITH Zeolite 